EPJ Web of Conferences 47, 05002 (2013)

DOI: $10.1051 /$ epjconf/20134705002

(C) Owned by the authors, published by EDP Sciences, 2013

\title{
Red Optical Planet Survey: A radial velocity search for low mass M dwarf planets
}

\author{
J.R. Barnes ${ }^{1, a}$, J.S. Jenkins ${ }^{2}$, H.R.A. Jones ${ }^{1}$, P. Rojo ${ }^{2}$, P. Arriagada ${ }^{3}$, \\ A. Jordán ${ }^{3}$, D. Minniti ${ }^{3,4}$, M. Tuomi ${ }^{1,5}$, S.V. Jeffers ${ }^{6}$ and D. Pinfield ${ }^{1}$ \\ ${ }^{1}$ Centre for Astrophysics Research, Univ. of Hertfordshire, Hatfield, UK \\ ${ }^{2}$ Departamento de Astronomía, Universidad de Chile, Santiago, Chile \\ ${ }^{3}$ Dept. of Astronomy and Astrophysics, Pontificia Universidad Catolica de Chile, \\ Santiago, Chile \\ ${ }^{4}$ Vatican Observatory, V00120 Vatican City State, Italy \\ ${ }^{5}$ University of Turku, Tuorla Observatory, Department of Physics and Astronomy, \\ Piikkiö, Finland \\ ${ }^{6}$ Institut für Astrophysik, Georg-August-Universität, Göttingen, Germany
}

\begin{abstract}
We present radial velocity results from our Red Optical Planet Survey (ROPS), aimed at detecting low-mass planets orbiting mid-late M dwarfs. The $\sim 10 \mathrm{~ms}^{-1}$ precision achieved over 2 consecutive nights with the MIKE spectrograph at Magellan Clay is also found on week long timescales with UVES at VLT. Since we find that UVES is expected to attain photon limited precision of order $2 \mathrm{~ms}^{-1}$ using our novel deconvolution technique, we are limited only by the $\left(\leq 10 \mathrm{~ms}^{-1}\right)$ stability of atmospheric lines. Rocky planet frequencies of $\eta_{\oplus}=0.3-0.7$ lead us to expect high planet yields, enabling determination of $\eta_{\oplus}$ for the uncharted mid-late $\mathrm{M}$ dwarfs with modest surveys.
\end{abstract}

\section{INTRODUCTION}

Despite comprising $70 \%$ of the solar neighbourhood population [1], the lower mass $\mathrm{M}$ dwarfs have remained beyond efficient detection with optical based surveys as the stellar flux peaks at longer wavelengths. At infrared wavelengths, surveys targeted at searching for low mass planetary systems associated with low mass stars can be achieved with realistic timescales. Nevertheless, to date, only one survey, using CRIRES [2], has reported sub-10 $\mathrm{ms}^{-1}$ precision, with $5.4 \mathrm{~ms}^{-1}$ reported using an $\mathrm{NH}_{3}$ gas cell and modest $364 \AA$ of spectrum in the K-band. More recent surveys have used telluric lines as a reference fiducial, and while [3] have obtained $180-300 \mathrm{~ms}^{-1}$ precision at $\mathrm{R} \sim 20,0000$, [4] have achieved $\sim 50 \mathrm{~ms}^{-1}$ precision which is limited by activity on their sample of young active stars. Equally, the red-optical regime offers similar advantages with a $\mathrm{V}-\mathrm{I} \sim 2-5$ for the earliest, through to the latest $\mathrm{M}$ dwarfs respectively, but with much improved information content compared with the near-infrared $\mathrm{K}$ band wavelengths [2].

\section{PRECISION RADIAL VELOCITIES IN THE RED-OPTICAL}

In 2010 November $21 \& 22$, we observed with MIKE at Magellan Clay, while more recent observations spanning 6 half nights, from 2012 August 22-28 were made with UVES at VLT. The MIKE

\footnotetext{
${ }^{a}$ e-mail: j.r.barnes@herts.ac.uk
}

This is an Open Access article distributed under the terms of the Creative Commons Attribution License 2.0, which permits unrestricted use, distribution, and reproduction in any medium, provided the original work is properly cited. 


\section{EPJ Web of Conferences}

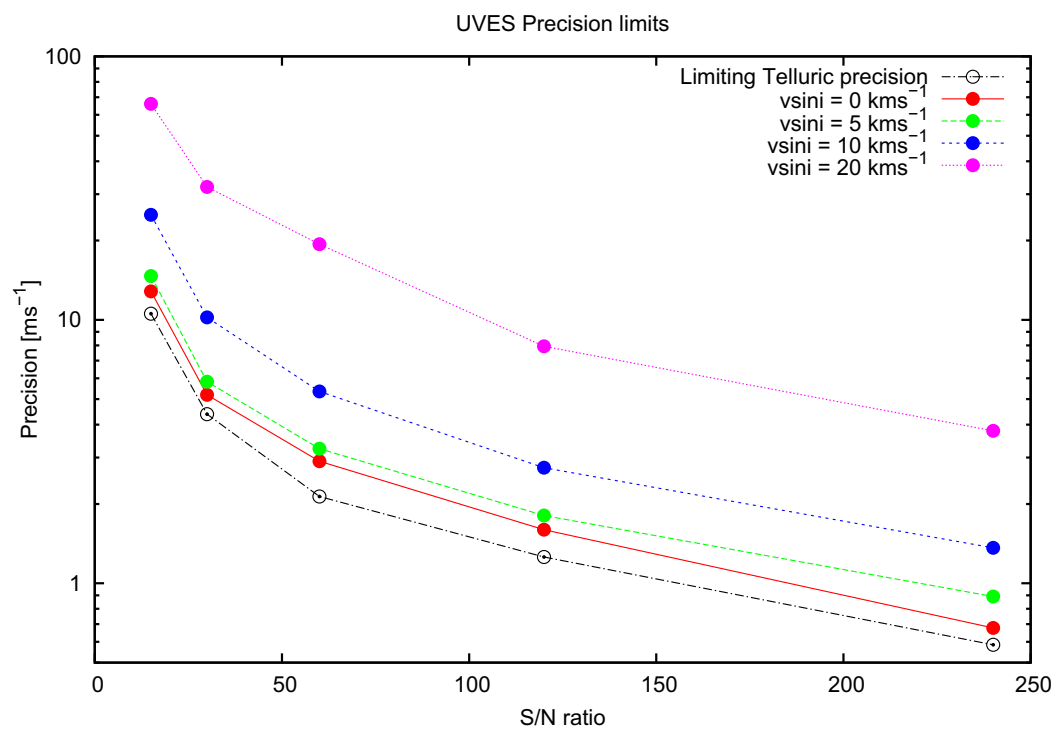

Figure 1. Simulated photon noise limited precision in the red-optical for $0.65-1.03 \mu \mathrm{m}$ with $\mathrm{R}=50 \mathrm{k}$. The simulation includes both the stellar and telluric line contributions. The curves are plotted for stellar rotation of $v \sin i=0,5,10 \& 20 \mathrm{kms}^{-1}$. Stellar synthetic spectra with $\mathrm{T}=2800 \mathrm{~K}$ were used [9] while synthetic telluric spectra are generated using the Line By Line Radiative Transfer Model code [10, 11], which is distributed by Atmospheric and Environmental Research (http://rtweb.aer.com).

observations spanned $0.63-0.89 \mu \mathrm{m}$ at a resolution of $\mathrm{R} \sim 35 k$, while UVES afforded $0.65-1.03 \mu \mathrm{m}$ and a higher resolution of $\mathrm{R} \sim 50 \mathrm{k}$. Least squares deconvolution is employed [5], enabling simultaneous and independent high $\mathrm{S} / \mathrm{N}$ ratio line profiles (typically of order several thousand) to be derived for the stellar and telluric lines. Deconvolution is especially apt for $\mathrm{M}$ dwarf spectra that have crowded lines, as blending effects are removed. With no simultaneous reference fiducial currently available at red-optical wavelengths, the telluric lines are employed, which have proven stability at the $10 \mathrm{~ms}^{-1}$ level [6], with a more recent study demonstrating sub- $10 \mathrm{~ms}^{-1}$ precision is possible [7]. Corrected radial velocities are thus directly determined by differencing the stellar and telluric line positions (obtained via crosscorrelation of the deconvolved line profiles) and application of heliocentric corrections. A fuller account of the methods are presented in [8], where a $3 \mathrm{~ms}^{-1}$ photon noise limited precision is estimated for MIKE. Here, in Fig. 1 we show that the extended wavelength coverage and higher resolution of UVES indicates a $2 \mathrm{~ms}^{-1}$ precision limit for $\mathrm{S} / \mathrm{N} \sim 100$ and a stellar $v \sin i=5 \mathrm{kms}^{-1}$.

\section{RESULTS AND DISCUSSION}

In Fig. 2 we show results from a selection of our target sample for MIKE (left panels) and UVES (right panels). A total of 7 targets were observed with MIKE and are presented in full in [8]. Large shifts of up to 1 pixel (equivalent to $\sim 2150 \mathrm{~ms}^{-1}$ ) were seen in the ThAr lines indicating that the Nasmyth mounted spectrograph was subject to mechanical instabilities. It is unclear whether such shifts result from repeated calibration frame measurements or from gravitational settling of the dewar. Nevertheless, the simultaneously derived telluric and stellar line positions should largely remove these effects, in addition to any reddening due to the changing slit angle on the sky. UVES suffers less from these effects and shows drifts of only a few $100 \mathrm{~ms}^{-1}$ throughout the night which correlate well with temperature within the spectrograph. It seems likely that the increased wavelength coverage and stability contributes to the improved precision in the UVES data set. Overall precision of sub- $5 \mathrm{~ms}^{-1}$ precision is found 


\section{Hot Planets and Cool Stars}

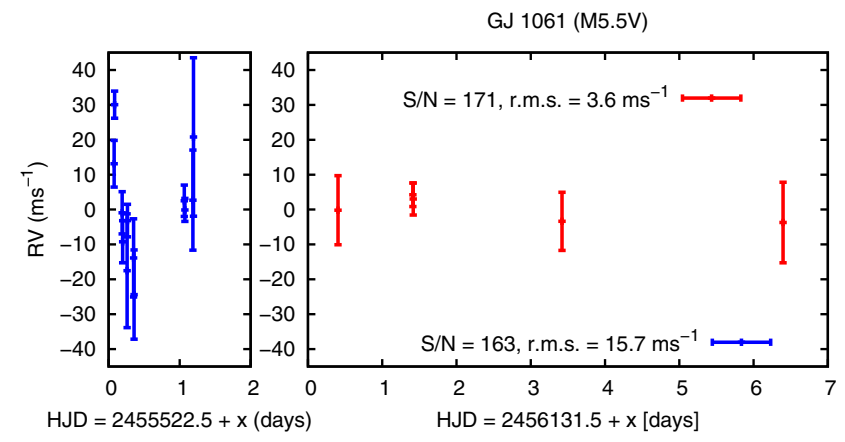

GJ 1002 (M5.5V)
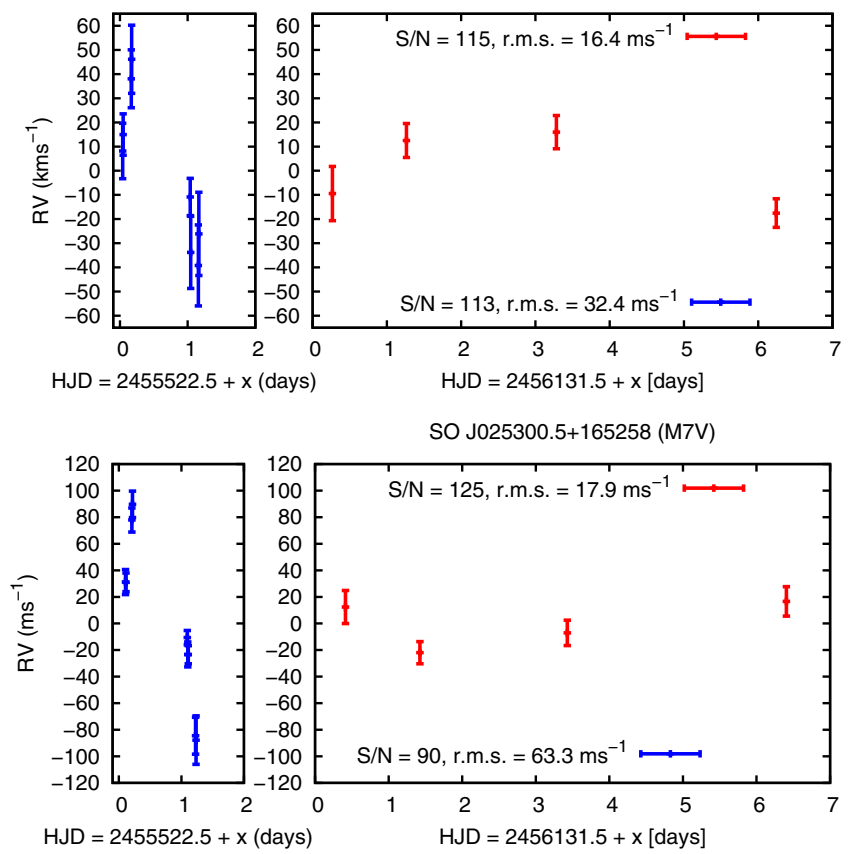

Figure 2. Heliocentric corrected radial velocities for GJ 1061 (M5.5V), GJ 1002 (M5.5V) and SO J025300 + 165258 (M7V). The left panel shows the 2010 Nov 21 \& 22 velocities derived from MIKE while the right panel shows the 2012 Jul $22-28$ velocities from UVES. The mean extracted S/N ratios of the spectra used to derive the radial velocities are given. Deconvolution results in a single stellar line profile per spectrum $(\mathrm{S} / \mathrm{N}=7800-11500$ for these objects) and a single telluric line per spectrum $(\mathrm{S} / \mathrm{N}=3000-4500)$ enabling precise radial velocities to be measured via cross-correlation. The MIKE data possess r.m.s. scatter of $\sim 2-4$ times that seen in the UVES data.

for GJ 1061 (input $\mathrm{S} / \mathrm{N}=170$, deconvolved $\mathrm{S} / \mathrm{N}$ 10,500-11,500). Further observations, possibly with sub-day sampling, are required to determine whether the $P=2.06 \mathrm{~d}, K_{*}=182.7 \mathrm{kms}^{-1}$ signal, found at 6.4- $\sigma$ in the MIKE data [8] is also present in the UVES data.

Our complete sample includes M5.5V-M9V objects with $m_{I}=9-14.5$, of which up to 30 can be observed in a full night. For fainter targets our precision is limited by both $\mathrm{S} / \mathrm{N}$ and stellar rotation. For an M6V observed with $m_{I}=13-14$, and typical $v \sin i=10 \mathrm{kms}^{-1}$, we achieve $\mathrm{S} / \mathrm{N}$ ratios of $30-50$ in $1200 \mathrm{~s}$, yielding respective velocity precisions of $\sim 13-19 \mathrm{~ms}^{-1}$. Habitable zone planets, with equivalent velocity amplitudes will possess lower mass limits of $7-11 \mathrm{M}_{\oplus}$. Similarly, for a M9V observed with $m_{I}=14.5$, and typical $v \sin i=15 \mathrm{kms}^{-1}$, we expect to achieve $\mathrm{S} / \mathrm{N} \sim 25$ in $1800 \mathrm{~s}$, 
yielding a velocity precision of $\sim 36 \mathrm{~ms}^{-1}$. For habitable zone planets, this corresponds to lower mass limits of $13 \mathrm{M}_{\oplus}\left(0.77 \mathrm{M}_{\text {Neptune }}\right)$. We note that there are still many stars with $v \sin i \leq 5 \mathrm{kms}^{-1}$, enabling precisions of $\leq 10 \mathrm{~ms}^{-1}$ to be achieved, equivalent to planetary masses as low as $5 \mathrm{M}_{\oplus}$.

Kepler planetary candidates now confirm that Earth-mass to Neptune-mass planets are $\sim 2.5-4$ times more numerous around M stars than earlier spectral types [12], and crucially are found in greater numbers than transiting Jupiter size planets orbiting earlier spectral types. Many of these candidates are in in very close orbits of $\lesssim 0.1$ AU. Moreover, the frequency of habitable planets $\left(1 \mathrm{M}_{\oplus}<m \sin i<\right.$ $10 \mathrm{M}_{\oplus}$ ) orbiting early $\mathrm{M}$ dwarfs has been estimated at $\eta_{\oplus}=0.41_{-0.13}^{+0.54}$ [13]. By scaling the increased $\mathrm{M}$ dwarf frequency of rocky planet candidates in the Kepler survey with the findings of radial velocity surveys at earlier spectral types [14], we estimate that 30-70\% of M dwarfs are expected to host rocky planets. By a similar argument, we expect $13-27 \%$ of $\mathrm{M}$ dwarfs to harbour Neptune-mass planets. Hence, assuming that these estimations can be extrapolated to mid-late $\mathrm{M}$ dwarfs, it is expected that small survey samples should return a high yield of low-mass planet detections.

\section{References}

[1] T.J. Henry, P.A. Ianna, J.D. Kirkpatrick, H. Jahreiss, AJ 114, 388 (1997)

[2] J.L. Bean, A. Seifahrt, H. Hartman, H. Nilsson, G. Wiedemann, A. Reiners, S. Dreizler, T.J. Henry, ApJ 713, 410 (2010), 0911.3148

[3] F. Rodler, R. Deshpande, M.R. Zapatero Osorio, E.L. Martín, M.M. Montgomery, C. Del Burgo, O.L. Creevey, A\&A 538, A141 (2012), 1112.1382

[4] J.I. Bailey, III, R.J. White, C.H. Blake, D. Charbonneau, T.S. Barman, A.M. Tanner, G. Torres, ArXiv e-prints (2012), 1202.0300

[5] J.R. Barnes, A. Collier Cameron, Y.C. Unruh, J.F. Donati, G.A.J. Hussain, MNRAS 299, 904 (1998)

[6] D.F. Gray, K.I.T. Brown, PASP 118, 399 (2006)

[7] P. Figueira, F. Pepe, C. Lovis, M. Mayor, A\&A 515, A106 (2010), 1003.0541

[8] J.R. Barnes, J.S. Jenkins, H.R.A. Jones, P. Rojo, P. Arriagada, A. Jordán, D. Minniti, M. Tuomi, S.V. Jeffers, D. Pinfield, MNRAS 424, 591 (2012), 1204.6283

[9] I. Brott, P.H. Hauschildt, A PHOENIX Model Atmosphere Grid for Gaia, in The ThreeDimensional Universe with Gaia, edited by C. Turon, K.S. O'Flaherty, \& M.A.C. Perryman (2005), Vol. 576 of ESA Special Publication, pp. 565-+

[10] S.A. Clough, M.J. Iacono, J.L. Moncet, J. Geophys. Res. 97, 15761 (1992)

[11] S.A. Clough, S.M. W., E.J. Mlawer, J.S. Delamere, M.J. Iacono, K. Cady-Pereira, S. Boukabara, P. Brown, J. Quant. Spectrosc. Radiat. Transfer 91, 233 (2005)

[12] W.J. Borucki, D.G. Koch, G. Basri, N. Batalha, T.M. Brown, S.T. Bryson, D. Caldwell, J. Christensen-Dalsgaard, W.D. Cochran, E. DeVore et al., ApJ 736, 19 (2011), 1102.0541

[13] X. Bonfils, X. Delfosse, S. Udry, T. Forveille, M. Mayor, C. Perrier, F. Bouchy, M. Gillon, C. Lovis, F. Pepe et al., ArXiv: 1111.5019 (2011), 1111.5019

[14] A.W. Howard, G.W. Marcy, J.A. Johnson, D.A. Fischer, J.T. Wright, H. Isaacson, J.A. Valenti, J. Anderson, D.N.C. Lin, S. Ida, "Science” 330, 653 (2010), 1011.0143 